\title{
A compressible boundary layer optimal control approach using nonlinear boundary region equations
}

\author{
Omar Es-Sahli *1, Adrian Sescu ${ }^{\dagger 1}$, Mohammed Afsar ${ }^{\ddagger 2}$, Yuji Hattori ${ }^{\S 3}$, and Makoto \\ Hirota $^{\llbracket 3}$ \\ ${ }^{1}$ Department of Aerospace Engineering, Mississippi State University, MS 39762 \\ ${ }^{2}$ Department of Mechanical \& Aerospace Engineering, Strathclyde University, 75 Montrose \\ St. Glasgow, G1 1XJ, UK \\ ${ }^{3}$ Institute of Fluid Science, Tohoku University, 2 Chome-1-1 Katahira, Aoba Ward, Sendai, \\ 980-8577, Japan
}

\begin{abstract}
High-amplitude free-stream turbulence and large surface roughness elements can excite a laminar boundary layer sufficiently enough to cause streamwise oriented vortices to form. The latter is accompanied by streaks of varying amplitudes that 'wobble' through inviscid secondary instabilities and, ultimately, transition to turbulence. In this paper, we formulate a mathematical framework for the optimal control of compressible boundary layers to suppress the growth rate of the streamwise vortex system before breakdown occurs. This has a commensurate impact on the wall shear stress and heat transfer at the wall. Flow instabilities are introduced either through roughness elements equally separated in the spanwise direction or via free-stream disturbances. The compressible Navier-Stokes equations are reduced to the boundary region equations (BRE) in a high Reynolds number asymptotic framework wherein the streamwise wavelengths of the disturbances are assumed to be much larger than the spanwise and wall-normal counterparts. The method of Lagrange multipliers is used to derive the adjoint compressible boundary region equations via an appropriate transformation of the original constrained optimization problem into an unconstrained form. In the present formulation, the wall transpiration velocity represents the control variable while the wall shear stress or the vortex energy represents the cost functional. Our study shows that this kind of control approach induces a significant reduction in the kinetic energy and wall shear stress of the boundary layer flow. Contour plots visually demonstrate how the primary instabilities gradually flatten out as more control iterations are applied.
\end{abstract}

\section{Introduction}

Flow control techniques are an important aspect of fluid mechanical research because of the possible optimization and improvement in the performance and operation of a fluid system. For example, automobiles, aircraft and marine vehicles all offer implementation of such control methods to determine suitable configuration of wings surface/edge geometry, engine inlet and nozzle design etc. to improve a set of design metrics. The metric could be the increase in the lift to drag ratio on the aerodynamic surface or to delay/accelerate the transition to turbulence point to postpone flow separation, enhance mixing or alter the morphology of the vortices embedded within the turbulence field.

Controlling transitional or fully-developed turbulent boundary layers is mainly intended to achieve a reduction in the energy carried by vortex structures that are aligned in the streamwise direction. These take the form of alternate high- and low-velocity streaks originating in the near-wall region (e.g., Kendall, ${ }^{24}$

\footnotetext{
*Research Assistant, Department of Aerospace Engineering, Mississippi State University, MS 39762; member AIAA.

${ }^{\dagger}$ Associate Professor, Department of Aerospace Engineering, Mississippi State University, MS 39762; Associate Fellow AIAA.

$\ddagger$ Chancellor Fellow, Department of Mechanical \& Aerospace Engineering; AIAA member.

$\S$ Professor, Institute of Fluid Science, Tohoku University.

$\mathbb{I}$ Assistant Professor, Institute of Fluid Science, Tohoku University.
} 
Matsubara \& Alfredsson, ${ }^{38}$ or Landahl ${ }^{28}$ ). For boundary layers over flat plates or wings, such streaky flows appear when the upstream roughness height is greater than a critical value (e.g., Choudhari \& Fischer, ${ }^{6}$ White, ${ }^{56}$ White et al. ${ }^{57}$ Goldstein et al. ${ }^{12-14}$ or Wu \& Choudhari $\left.{ }^{59}\right)$, or when the amplitude of the freestream disturbances exceeds a certain threshold (e.g., Kendall, ${ }^{24}$ Westin et al.,${ }^{55}$ Matsubara \& Alfredsson, ${ }^{38}$ Leib et al. ${ }^{30}$ Zaki \& Durbin, ${ }^{62}$ Goldstein \& Sescu, ${ }^{15}$ or Ricco et al. $\left.{ }^{43}\right)$. For the boundary layer flow along a concave surface, the streamwise elongated streaks are referred to as Görtler vortices and they develop as a result of the imbalance between the centrifugal forces and the wall-imposed pressure gradient (e.g., Gortler, ${ }^{16}$ Hall, ${ }^{18-20}$ Swearingen \& Blackwelder, ${ }^{54}$ Malik \& Hussaini, ${ }^{36}$ Saric, ${ }^{45}$ Li \& Malik,${ }^{31}$ Boiko et al., ${ }^{2}$ Wu et al., ${ }^{58}$ or Sescu et al., ${ }^{47,48}$ Es-Sahli et al., ${ }^{9}$ Ren \& Fu ${ }^{42}$ Dempsey et al., ${ }^{8}$ Xu et al. ${ }^{61}$ ). The higher the wall curvature, the more rapid the vortex formation is and transition. Similarly, small to medium wall curvature also induces vortex formation however; the latter in both instances alters the primary flow and causes the laminar flow to breakdown.

Like many other types of instabilities, streamwise-oriented vortices and their accompanying streaks are important in various engineering applications such turbo-machinery blades, and the flow in the proximity to the walls of wind tunnels or turbofan engine intakes. Not only do these instabilities result in transition, but there is also a significant increase in noise for supersonic and hypersonic wind tunnels and this can cause interference with the measurements at the test section (Schneider ${ }^{46}$ ). This makes it a challenging task to compare between wind tunnel measurements and real flight conditions. The objective function for a control algorithm in this context would be to lessen streamwise vortex energy, which is expected to delay nonlinear breakdown and the transition. But since the transient part of the primary instability is responsible for the growth of three-dimensional disturbances (and subsequent breakdown) the control strategy must focus on restricting the growth of the transient modes.

Although significant achievements have been made in the last decades toward understanding the phenomenology behind the formation and the influence of the streamwise vortices and streaks in incompressible boundary layers, the progress in the compressible regime has been slow and bypass transition at high upstream flow speeds remains largely unexplored. This work is directed towards delving into this parametric regime to further investigate the effects of wall transpiration within a self-consistent asymptotic mathematical framework.

Wall transpiration control techniques of boundary layers can be applied via localized suction and blowing regions, respectively, underneath low- and high-velocity streaks. The net result is a decrease in the span-wise variation of the streamwise velocity and, therefore, a corresponding reduction in the number and strength of the bursting events.

Alternatively, this can be achieved through active wall control, which has been utilized in the context of turbulent channel flow (see Choi, Moin \& $\mathrm{Kim}^{5}$ ) as a technique to reduce skin friction drag. Choi et al. ${ }^{5}$ conducted direct numerical simulations using active wall control based on wall transpiration, by placing sensors in a sectional plane that is parallel to the wall; the technique achieved approximately $25 \%$ reduction in frictional drag. Practically speaking, it is very challenging and difficult, if not impossible, to install sensors in the flow as they may amplify existing disturbances, or induce new ones. Thus, to avoid interference issues, Choi et al. ${ }^{5}$ investigated the same control algorithm but with sensors placed at the wall with information based on the leading term in the Taylor series expansion of the vertical component of velocity near the wall; however, this approach resulted only in a $6 \%$ reduction. Koumoutsakos ${ }^{26,27}$ implemented a similar feedback control algorithm informed by flow quantities at the wall. Taking vorticity flux components as inputs to the control algorithm resulted in a more significant skin friction reduction (approximately 40\%).

Lee et al. ${ }^{29}$ derived new suboptimal feedback control laws based on blowing and suction to manipulate the flow structures in the proximity to the wall using surface pressure or shear stress distribution (the reduction in the frictional drag was in the range of 16-20\%). Observing that the 'opposition control' technique is more effective at low Reynolds number turbulent wall flows, Pamies et al. ${ }^{40}$ proposed the utilization of blowing only at high Reynolds numbers, and by doing so they obtained significant reduction in the skin-friction drag for these flows. Recently, Stroch et al. ${ }^{53}$ conducted a comparison between the opposition control applied in the framework of turbulent channel flow and a spatially developing turbulent boundary layer. They found that the rates of frictional drag reduction are approximately similar in both cases. An overview of the issues and limitations associated with the opposition control type is given in the review article by Kim. ${ }^{25}$

Hogberg et al. ${ }^{21}$ reported the first successful re-laminarization of a $R e_{\tau}=100$ turbulent channel flow by applying zero mass flux blowing and suction at the wall in the framework of linear full-state optimal control theory. They showed that the information available in the linearized equations may be sufficient to construct 
linear controllers able to re-laminarize a wall turbulent flow, but this may be limited to low Reynolds number flows.

A number of experiments aiming to control disturbances in laminar or turbulent boundary layers by blowing and suction have been conducted over the years. Several of them are briefly mentioned here. Gad-el-Hak \& Blackwelder ${ }^{11}$ used continuous or intermittent suction to eliminate artificially generated disturbances in a flat-plate boundary layer. The same idea was used in the experiments conducted by Myose \& Blackwelder ${ }^{39}$ to delay the breakdown of Görtler vortices. Jacobson \& Reynolds ${ }^{22}$ developed a new type of actuation based on a vortex generator to control disturbances generated by a cylinder with the axis normal to the wall, and unsteady boundary layer streaks generated by pulsed suction. Regarding the latter, the actuation was able to significantly reduce the spanwise gradients of the streamwise velocity, which are known to be an important driving force of secondary instabilities (see Swearingen \& Blackwelder ${ }^{54}$ ). In the experiments of Lundell \& Alfredsson, ${ }^{35}$ streamwise velocity streaks in a channel flow were controlled by localized regions of suctions in the downstream, which are found to be effective in delaying secondary instabilities and consequently the transition onset.

Optimal control in the framework of laminar or turbulent boundary layers has been utilized in a number of studies. There are numerous studies pertaining the application of optimal control of shear flows (see the review of Gunzburger ${ }^{17}$ or a more recent review of Luchini \& Bottaro, ${ }^{34}$ although the latter is in a slightly different context). The following studies have targeted the control of disturbances evolving in laminar or turbulent boundary layers (e.g..$\left.^{1,3,4,7,21,23,33,51,52,63}\right)$.

Optimal control of turbulent channel flows by blowing and suction was employed previously by Bewley \& Moin, ${ }^{1}$ who claimed a $17 \%$ frictional drag reduction as a result of this scheme. Blowing and suction based optimal control was also applied by Cathalifaud \& Luchini ${ }^{3}$ to reduce the energy of disturbances in a flatplate and a concave boundary layer. In the study of Zuccher et al. ${ }^{63}$ an optimal and robust control strategy was discussed and tested in the framework of steady three-dimensional disturbances (in the form of streaks) that form in a flat-plate boundary layer. It was based on an adjoint-based optimization technique to first find the optimal state for given initial conditions, and then to determine what the worst initial conditions for the optimal control are. Lu et al. ${ }^{33}$ derived an optimal control scheme within the linearized unsteady boundary region equations which are the asymptotic reduction of the Navier-Stokes equations under the assumption of low frequency and low streamwise wavelength. Their study aimed at controlling both streaks developing in flat-plate boundary layers and Görtler vortices evolving along concave surfaces. Cherubini et al. ${ }^{4}$ applied a nonlinear optimal control strategy with blowing and suction, starting with the full Navier-Stokes equations, and using the method of Lagrange multipliers to determine the largest decrease of the disturbance energy.

A closed-loop optimal control technique based on wall transpiration was derived and tested by Papadakis, $\mathrm{Lu} \&$ Ricco ${ }^{41}$ in the framework of a flat-plate laminar boundary layer excited by freestream disturbances. The optimal control was split into two sequences that can be obtained by marching the corresponding equations in forward and backward directions; it was found that the feedback sequence is more important than the feed-forward sequence. The study of Xiao \& Papadakis ${ }^{60}$ employs an optimal control algorithm based on Lagrange multipliers, aimed at delaying transition in a flat-plate boundary layer excited by freestream vortical disturbances, is based on blowing and suction, and is derived in the framework of full Navier-Stokes equations.

The present paper is an extension of the work of Sescu \& Afsar ${ }^{51}$ to the compressible regime. They implemented an optimal control approach to limit the growth of Görtler vortices developing in an incompressible laminar boundary layer flow over a concave wall. They derived the adjoint equation and the associate optimality condition based on the incompressible boundary region equations and employed wall deformation and velocity transpiration as flow control parameters. They showed that that the optimal control algorithm is very effective in reducing the amplitude of the Görtler vortices, especially for the control based on wall displacement. Here, we derive the adjoint equations and the optimality condition for the compressible boundary region equations, which represent the high Reynolds number extension of the full compressible Navier-Stokes equations. The control variable is represented by the transpiration velocity, while the wall shear stress or the vortex energy represents the cost functional. Results involving linear Görtler vortices are reported for high supersonic Mach numbers, for which the control algorithm was found to be stable. 


\section{Basic scalings and governing equations}

\section{A. Scalings}

All dimensional spatial coordinates $\left(x^{*}, y^{*}, z^{*}\right)$ are normalized by the spanwise separation $\lambda^{*}$, while the dependent variables by their respective freestream values, except the pressure, which is normalized by the dynamic pressure:

$$
\begin{gathered}
\bar{t}=\frac{t^{*}}{\lambda^{*} / V_{\infty}^{*}} ; \quad \bar{x}=\frac{x^{*}}{\lambda^{*}} ; \quad \bar{y}=\frac{y^{*}}{\lambda^{*}} ; \quad \bar{z}=\frac{z^{*}}{\lambda^{*}} \\
\bar{u}=\frac{u^{*}}{V_{\infty}^{*}} ; \quad \bar{v}=\frac{v^{*}}{V_{\infty}^{*}} ; \quad \bar{w}=\frac{w^{*}}{V_{\infty}^{*}} ; \quad \bar{\rho}=\frac{\rho^{*}}{\rho_{\infty}^{*}} \\
\bar{p}=\frac{p^{*}-p_{\infty}^{*}}{\rho_{\infty}^{*} V_{\infty}^{* 2}} ; \quad \bar{T}=\frac{T^{*}}{T_{\infty}^{*}} ; \quad \bar{\mu}=\frac{\mu^{*}}{\mu_{\infty}^{*}} ; \quad \bar{k}=\frac{k^{*}}{k_{\infty}^{*}}
\end{gathered}
$$

where $\lambda^{*}$ is the spanwise wavelength of the disturbances, $\left(u^{*}, v^{*}, w^{*}\right)$ are the velocity components, $\rho^{*}$ the density, $p^{*}$ is pressure, $T^{*}$ temperature, $\mu^{*}$ dynamic viscosity, $k^{*}$ thermal conductivity, and all quantities with $\infty$ at the subscript represent conditions at infinity.

Reynolds number based on the spanwise separation, Mach number and Prandtl number are defined as

$$
R_{\lambda}=\frac{\rho_{\infty}^{*} V_{\infty}^{*} \lambda^{*}}{\mu_{\infty}^{*}}, \quad M a=\frac{V_{\infty}^{*}}{a_{\infty}^{*}}, \quad \operatorname{Pr}=\frac{\mu_{\infty}^{*} C_{p}}{k_{\infty}^{*}}
$$

where $\mu_{\infty}^{*}, a_{\infty}^{*}$ and $k_{\infty}^{*}$ are freestream dynamic viscosity, speed of sound and thermal conductivity, respectively, and $C_{p}$ is the specific heat at constant pressure. For boundary layer flows over curved surfaces, we define the global Görtler number as

$$
G_{\lambda}=\frac{R_{\lambda}^{2} \lambda^{*}}{r^{*}}
$$

where $r^{*}$ is the radius of the curvature.

\section{B. Compressible Navier-Stokes equations (N-S)}

For a full compressible flow, the primitive form of the Navier-Stokes equations in non-dimensional variables are written as

$$
\begin{gathered}
\frac{D \bar{\rho}}{D t}+\rho\left(\frac{\partial \bar{u}}{\partial \bar{x}}+\frac{\partial \bar{v}}{\partial \bar{y}}+\frac{\partial \bar{w}}{\partial \bar{z}}\right)=0 \\
\bar{\rho} \frac{D \bar{u}}{D \bar{t}}=-\frac{\partial \bar{p}}{\partial \bar{x}}+\frac{1}{R e_{\lambda}} \frac{\partial}{\partial \bar{x}}\left[\frac{2}{3} \mu\left(2 \frac{\partial \bar{u}}{\partial \bar{x}}-\frac{\partial \bar{v}}{\partial \bar{y}}-\frac{\partial \bar{w}}{\partial \bar{z}}\right)\right]+\frac{\partial}{\partial \bar{y}}\left[\mu\left(\frac{\partial \bar{u}}{\partial \bar{y}}+\frac{\partial \bar{v}}{\partial \bar{x}}\right)\right]+\frac{\partial}{\partial \bar{z}}\left[\mu\left(\frac{\partial \bar{w}}{\partial \bar{x}}+\frac{\partial \bar{u}}{\partial \bar{z}}\right)\right] \\
\bar{\rho} \frac{D \bar{v}}{D \bar{t}}=-\frac{\partial \bar{p}}{\partial \bar{y}}+\frac{1}{R e_{\lambda}} \frac{\partial}{\partial \bar{y}}\left[\frac{2}{3} \mu\left(2 \frac{\partial \bar{v}}{\partial \bar{y}}-\frac{\partial \bar{u}}{\partial \bar{x}}-\frac{\partial \bar{w}}{\partial \bar{z}}\right)\right]+\frac{\partial}{\partial \bar{x}}\left[\mu\left(\frac{\partial \bar{v}}{\partial \bar{x}}+\frac{\partial \bar{u}}{\partial \bar{y}}\right)\right]+\frac{\partial}{\partial \bar{z}}\left[\mu\left(\frac{\partial \bar{v}}{\partial \bar{z}}+\frac{\partial \bar{w}}{\partial \bar{y}}\right)\right] \\
\bar{\rho} \frac{D \bar{w}}{D \bar{t}}=-\frac{\partial \bar{p}}{\partial \bar{z}}+\frac{1}{R e_{\lambda}} \frac{\partial}{\partial \bar{z}}\left[\frac{2}{3} \mu\left(2 \frac{\partial \bar{w}}{\partial \bar{z}}-\frac{\partial \bar{u}}{\partial \bar{x}}-\frac{\partial \bar{v}}{\partial \bar{y}}\right)\right]+\frac{\partial}{\partial \bar{x}}\left[\mu\left(\frac{\partial \bar{w}}{\partial \bar{x}}+\frac{\partial \bar{u}}{\partial \bar{z}}\right)\right]+\frac{\partial}{\partial \bar{y}}\left[\mu\left(\frac{\partial \bar{v}}{\partial \bar{z}}+\frac{\partial \bar{w}}{\partial \bar{y}}\right)\right] \\
\bar{\rho} \frac{D \bar{T}}{D \bar{t}}=\frac{1}{P r R e_{\lambda}}\left[\frac{\partial}{\partial \bar{x}}\left(k \frac{\partial \bar{T}}{\partial \bar{x}}\right)+\frac{\partial}{\partial \bar{y}}\left(k \frac{\partial \bar{T}}{\partial \bar{y}}\right)+\frac{\partial}{\partial \bar{z}}\left(k \frac{\partial \bar{T}}{\partial \bar{z}}\right)\right] \\
-(\gamma-1) M_{\infty}^{2}\left[p\left(\frac{\partial \bar{u}}{\partial \bar{x}}+\frac{\partial \bar{v}}{\partial \bar{y}}+\frac{\partial \bar{w}}{\partial \bar{z}}\right)-\frac{2}{3} \mu\left(\frac{\partial \bar{u}}{\partial \bar{x}}+\frac{\partial \bar{v}}{\partial \bar{y}}+\frac{\partial \bar{w}}{\partial \bar{z}}\right)^{2}\right] \\
+(\gamma-1) M_{\infty}^{2} \frac{\mu}{R e_{\lambda}}\left[2\left(\frac{\partial \bar{u}}{\partial \bar{x}}\right)^{2}+2\left(\frac{\partial \bar{v}}{\partial \bar{y}}\right)^{2}+2\left(\frac{\partial \bar{w}}{\partial \bar{z}}\right)^{2}+\left(\frac{\partial \bar{u}}{\partial \bar{y}}+\frac{\partial \bar{v}}{\partial \bar{x}}\right)^{2}+\left(\frac{\partial \bar{w}}{\partial \bar{x}}+\frac{\partial \bar{u}}{\partial \bar{z}}\right)^{2}+\left(\frac{\partial \bar{v}}{\partial \bar{z}}+\frac{\partial \bar{w}}{\partial \bar{y}}\right)^{2}\right]
\end{gathered}
$$


where

$$
\frac{D}{D \bar{t}}=\frac{\partial}{\partial \bar{t}}+\bar{u} \frac{\partial}{\partial \bar{x}}+\bar{v} \frac{\partial}{\partial \bar{y}}+\bar{w} \frac{\partial}{\partial \bar{z}}
$$

is the substantial derivative (for what follows, we consider the steady-state case for the N-S equations, i.e. $\partial / \partial \bar{t}=0)$. The pressure $p$, the temperature $T$ and the density of the fluid are combined in the equation of state in non-dimensional form, $\bar{p}=\bar{\rho} \bar{T} / \gamma M_{\infty}^{2}$, assuming that non-chemically-reacting flows are considered. Other notations include the dynamic viscosity $\mu$, Reynolds number $R e=\rho_{\infty} V_{\infty}^{*} \lambda^{*} / \mu$ based on a characteristic velocity $V_{\infty}^{*}$, and a characteristic length $L^{*}$, the free-stream Mach number $M_{\infty}=V_{\infty}^{*} / a_{\infty}^{*}$. The dynamic viscosity and thermal conductivity $k$ is linked to the temperature using the Sutherland's equations in dimensionless form,

$$
\mu=T^{b} ; \quad k=\frac{C_{p} \mu}{\operatorname{Pr}}
$$

where $b=0.76\left(\right.$ Ricco \& $\left.\mathrm{Wu}^{44}\right), C_{1}=1.458 \times 10^{-6}, C_{2}=110.4, C_{p}=\gamma R /(\gamma-1), \gamma=1.4$, and $\operatorname{Pr}=0.72$ for air.

\section{Compressible Nonlinear Boundary-Region Equations (BRE)}

We consider a compressible flow of uniform velocity $V_{\infty}^{*}$ and temperature $T_{\infty}^{*}$ past a flat or curved surface. The air is treated as a perfect gas so that the sound speed in the free-stream $c_{\infty}^{*}=\sqrt{\gamma R T_{\infty}^{*}}$, where $\gamma=1.4$ is the ratio of the specific heats, and $R=287.05 \mathrm{Nm} /(\mathrm{kgK})$ is the universal gas constant; Mach number is assumed to be of order one. The asymptotic structure of the flow is composed of four regions as in Leib et al., ${ }^{30}$ Ricco \& $\mathrm{Wu}^{43}$ or Marensi et al. ${ }^{37}$ (see figure 1 ):

- Region I in proximity to the the leading edge, where the flow is assumed inviscid and the disturbances are treated as small perturbations of the base flow.

- Region II is the boundary layer in the vicinity of the leading edge, with the thickness much smaller than the spanwise separation associated with the freestream disturbances

- Region III is the viscous region that follows in the downstream of region II; here, the boundary layer thickness is in the same order of magnitude as the spanwise separation

- Region IV is above region III, and the flow is assumed again inviscid since the viscous effects are negligible

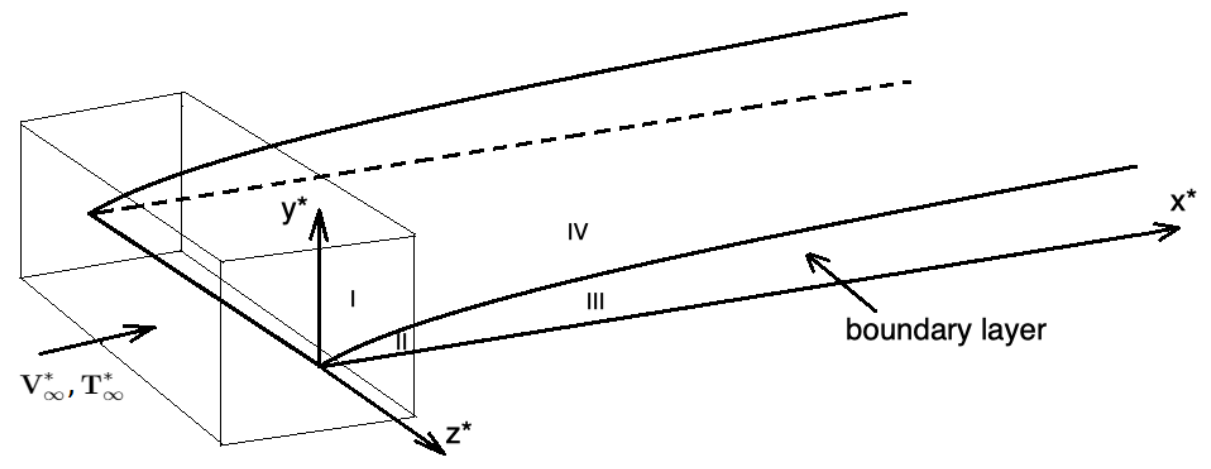

Figure 1: Illustration of the four regions composing the asymptotic structure of the flow.

The focus in this paper is the region III, where the streamwise wavenumber of disturbances are expected to be small, and the flow is governed by the boundary region equations. In this region, the compressible boundary region equations can be derived from the full steady-state compressible N-S equations. Based in the above assumption, the streamwise distance can be scaled as $x=\bar{x} / R_{\lambda}$ while the other two are the same 
$y=\bar{y}, z=\bar{z}$, and the time as $t=\bar{t} / R_{\lambda}$. Also, the crossflow components of velocity are expected to be small compared to the streamwise component, and variations of pressure are expected to be very small. This suggest the introduction of the scaling of dependent variables as:

$$
u=\bar{u} ; \quad v=\bar{v} / R_{\lambda} ; \quad w=\bar{w} / R_{\lambda} ; \quad \rho=\bar{\rho} ; \quad p=\bar{p} / R_{\lambda}^{2} ; \quad T=\bar{T} ; \quad \mu=\bar{\mu} ; \quad k=\bar{k}
$$

Plugging the scaled variables into the full steady-state N-S equations in curvilinear coordinates, and performing on order of magnitude analysis, we obtain the nonlinear compressible boundary region equations (NCBRE) in the form

$$
\begin{gathered}
\mathbf{V} \cdot \nabla \rho+\rho \nabla \cdot \mathbf{V}=0 \\
\rho \mathbf{V} \cdot \nabla u=\nabla_{c} \cdot\left(\mu \nabla_{c} u\right) \\
\rho \mathbf{V} \cdot \nabla v+G_{\lambda} u^{2}=-\frac{\partial p}{\partial y}+\frac{\partial}{\partial y}\left[\frac{2}{3} \mu\left(3 \frac{\partial v}{\partial y}-\nabla \cdot \mathbf{V}\right)\right]+\frac{\partial}{\partial x}\left(\mu \frac{\partial u}{\partial y}\right)+\frac{\partial}{\partial z}\left[\mu\left(\frac{\partial v}{\partial z}+\frac{\partial w}{\partial y}\right)\right] \\
\rho \mathbf{V} \cdot \nabla w=-\frac{\partial p}{\partial z}+\frac{\partial}{\partial z}\left[\frac{2}{3} \mu\left(3 \frac{\partial w}{\partial z}-\nabla \cdot \mathbf{V}\right)\right]+\frac{\partial}{\partial x}\left(\mu \frac{\partial u}{\partial z}\right)+\frac{\partial}{\partial y}\left[\mu\left(\frac{\partial v}{\partial z}+\frac{\partial w}{\partial y}\right)\right] \\
\rho \mathbf{V} \cdot \nabla T=\frac{1}{P r} \nabla_{c} \cdot\left(k \nabla_{c} T\right)+(\gamma-1) M_{\infty}^{2} \mu\left[\left(\frac{\partial u}{\partial y}\right)^{2}+\left(\frac{\partial u}{\partial z}\right)^{2}\right]
\end{gathered}
$$

where $\mathbf{V}$ is the velocity vector and $\nabla_{c}$ is the crossflow nabla operator:

$$
\mathbf{V}=u \vec{i}+v \vec{j}+w \vec{k} ; \quad \nabla_{c}=\frac{\partial}{\partial y} \vec{j}+\frac{\partial}{\partial z} \vec{k}
$$

The effect of the wall curvature is contained in the term involving the global Görtler number $G_{\lambda}$.

This set of equations is parabolic in the streamwise direction and elliptic in the spanwise direction. Appropriate initial/upstream and boundary conditions are necessary to close the problem; these conditions will be the same as those used by Ricco \& Wu. ${ }^{43}$ The NCBRE are solved efficiently using to the numerical algorithm developed in Es-Sahli et al. ${ }^{10}$

\section{Optimal control problem in the nonlinear regime}

While it is common for an optimal flow control problem to be formulated in the framework of a dynamical system (usually, described by a set of equations that are parabolic in time), here we replace the time direction by the $x$-direction owing to the parabolic character of the BRE in the streamwise direction, which necessitates streamwise marching to obtain a solution.

\section{A. Generic optimal control formalism}

To fix ideas, we first write equations (14)-(18) in the generic and more compact form

$$
\mathcal{G}(\mathbf{q})=0,
$$

for brevity, with initial and boundary conditions

$$
\begin{gathered}
\mathbf{q}(0, y, z)=\mathbf{q}_{0}(y, z) \\
\mathbf{q}(x, 0, z)=\phi, \quad \lim _{y \rightarrow \infty} \mathbf{q}(x, y, z)=\mathbf{q}_{B}(x, z),
\end{gathered}
$$

along the wall-normal direction, and periodic or symmetry boundary conditions in the span-wise direction, $z$. In equation $(20), \mathcal{G}()$ is the non-linear BRE differential operator in abstract notation, $\mathbf{q}=(\rho, u, v, w, T)$ is the vector of state variables, $\phi$ is the control variable associated with the boundary conditions (e.g., the transpiration velocity at the wall, $\left.v_{w}\right), \mathbf{q}_{0}(y, z)$ represents the upstream or initial condition in $x=0$, and 
$\mathbf{q}_{B}$ is a given function that specifies the boundary condition at infinity. We define an objective (or cost) functional as

$$
\mathcal{J}(\mathbf{q}, \phi)=\mathcal{E}(\mathbf{q})+\sigma\left(\left\|\phi_{x}\right\|^{\beta_{2}}+\|\phi\|^{\beta_{2}}\right),
$$

where $\mathcal{E}(\mathbf{q})$ is a specified target function to be minimized (e.g., the energy of the disturbance, or the wall shear stress; the latter is considered in this study), the second term on the right hand side of (23) is a penalization term depending on the norm of the control variable (usually, this quantity place a constraint on the magnitude of the admissible control variable, since it cannot increase or decrease indefinitely), $\sigma$ and $\beta$ are given constants, and subscript $x$ denotes derivative with respect to $x$. The norm \|\| in equation (23) is associated with an appropriate inner product of two complex functions, $f$ and $g$, defined as

$$
\langle f, g\rangle=\int_{0}^{X_{t}} f^{*} g d X
$$

in the space $\left[0, X_{t}\right]$, with $X_{t}$ being the terminal streamwise location (the star in (24) denotes complex conjugate).

A common approach to transform a (nonlinear) constrained optimization problem into an unconstrained problem is by using the method of Lagrange multipliers (see, for example, $,{ }^{23},{ }^{1763}$ ). To this end, we consider the Lagrangian

$$
\mathcal{L}\left(\mathbf{q}, \phi, \mathbf{q}^{a}\right)=\mathcal{J}(\mathbf{q}, \phi)-\left\langle\mathcal{G}(\mathbf{q}), \mathbf{q}^{a}\right\rangle,
$$

where $\mathbf{q}^{a}$ is the vector of Lagrange multipliers $\left(\rho^{a}, u^{a}, v^{a}, w^{a}, T^{a}\right)$, also known as the adjoint vector. In other words, the Lagrange multipliers are introduced in order to transform the minimization of $\mathcal{J}(\mathbf{q}, \phi)$ under the constraint $\mathcal{G}(\mathbf{q})=0$ into the unconstrained minimization of $\mathcal{L}\left(\mathbf{q}, \phi, \mathbf{q}^{a}\right)$. The unconstrained optimization problem can be formulated as:

Find the control variable $\phi$, the state variables $\boldsymbol{q}$, and the adjoint variables $\boldsymbol{q}^{a}$ such that the Lagrangian $\mathcal{L}\left(\boldsymbol{q}, \phi, \boldsymbol{q}^{a}\right)$ is a stationary function, that is

$$
\delta \mathcal{L}=\frac{\partial \mathcal{L}}{\partial \mathbf{q}} \delta \mathbf{q}+\frac{\partial \mathcal{L}}{\partial \phi} \delta \phi+\frac{\partial \mathcal{L}}{\partial \mathbf{q}^{a}} \delta \mathbf{q}^{a}=0
$$

where

$$
\frac{\partial \mathcal{L}}{\partial a} \delta a=\frac{\mathcal{L}(a+\epsilon \delta a)-\mathcal{L}(a)}{\epsilon}
$$

represents directional differentiation in the generic direction $\delta a$. All directional derivatives in (26) must vanish, providing different sets of equations:

- adjoint BRE equations are obtained by taking the derivative with respect to $\mathbf{q}$,

$$
\frac{\partial \mathcal{L}}{\partial \mathbf{q}}=0 \quad \Rightarrow \quad \mathcal{G}^{a}\left(\mathbf{q}^{a}\right)=0
$$

- optimality conditions are obtained by taking the derivatives with respect to $\phi$,

$$
\frac{\partial \mathcal{L}}{\partial \phi}=0 \quad \Rightarrow \quad \mathcal{O}\left(\mathbf{q}^{a}, \mathbf{q}, \phi\right)=0
$$

- the original BRE equations are obtained by taking the derivative with respect to $\mathbf{q}^{a}$,

$$
\frac{\partial \mathcal{L}}{\partial \mathbf{q}_{a}}=0 \quad \Rightarrow \quad \mathcal{G}(\mathbf{q}, \psi)=0
$$


Equations (28)-(30) form the optimal control system that can be utilized to determine the optimal states and the control variables. One can note that the stationarity of the Lagrangian with respect to the adjoint variables $\mathbf{q}^{a}=\left(\rho^{a}, u^{a}, v^{a}, w^{a}, T^{a}\right)$ essentially yields the original state equations, while the stationarity with respect to the state variables $\mathbf{q}=(\rho, u, v, w, T)$ yields the adjoint equations that depend on the state variables. The relationship between the state variables and the adjoint variables can be expressed by the adjoint identity,

$$
\left\langle\mathcal{G}(\mathbf{q}), \mathbf{q}^{a}\right\rangle=\left\langle\mathbf{q}, \mathcal{G}^{a}\left(\mathbf{q}^{a}\right)\right\rangle+\mathcal{B}(\phi)
$$

where the last term, $\mathcal{B}$, represents a residual from the boundary conditions.

\section{B. Adjoint compressible BRE}

In our particular case of a boundary layer flow over a flat or concave surface with wall transpiration, the adjoint compressible BRE are derived starting with the integral

$$
\begin{aligned}
\mathcal{L}( & \left.\rho, u, v, w, T, p, \mu, k, v_{w}, \rho^{a}, u^{a}, v^{a}, w^{a}, T^{a}, p^{a}, \mu^{a}, k^{a}, s\right) \\
& =\frac{\sigma}{2} \int_{X_{0}}^{X_{1}} \int_{\Gamma}\left\|\frac{\partial v_{w}}{\partial X}\right\|^{2}+\left\|v_{w}\right\|^{2} d \Gamma d X-\int_{0}^{X_{0}} \int_{\Gamma} s v d \Gamma d X-\int_{X_{0}}^{X_{1}} \int_{\Gamma} s\left(v-v_{w}\right) d \Gamma d X-\int_{X_{1}}^{X_{t}} \int_{\Gamma} s v d \Gamma d X \\
& +\frac{\alpha}{2} \int_{X_{s 0}}^{X_{s 1}} \int_{\Gamma}\left\|\tau_{w}-\tau_{0}\right\|^{2} d \Gamma d X \\
& -\int_{0}^{X_{t}} \int_{\Omega} \rho^{a}(\mathbf{V} \cdot \nabla \rho+\rho \nabla \mathbf{V}) d \Omega d X \\
& -\int_{0}^{X_{t}} \int_{\Omega} u^{a}\left[\rho \mathbf{V} \cdot \nabla u-\nabla_{c} \cdot\left(\mu \nabla_{c} u\right)\right] d \Omega d X \\
& -\int_{0}^{X_{t}} \int_{\Omega} v^{a}\left\{\rho \mathbf{V} \cdot \nabla v+G_{\Lambda} u^{2}+\frac{\partial p}{\partial y}-\frac{\partial}{\partial y}\left[\frac{2}{3} \mu\left(3 \frac{\partial v}{\partial y}-\nabla \cdot \mathbf{V}\right)\right]-\frac{\partial}{\partial x}\left(\mu \frac{\partial u}{\partial y}\right)-\frac{\partial}{\partial z}\left[\mu\left(\frac{\partial v}{\partial z}+\frac{\partial w}{\partial y}\right)\right]\right\} d \Omega d X \\
& -\int_{0}^{X_{t}} \int_{\Omega} w^{a}\left\{\rho \boldsymbol{V} \cdot \nabla w+\frac{\partial p}{\partial z}-\frac{\partial}{\partial z}\left[\frac{2}{3} \mu\left(3 \frac{\partial w}{\partial z}-\nabla \cdot \mathbf{V}\right)\right]-\frac{\partial}{\partial x}\left(\mu \frac{\partial u}{\partial z}\right)-\frac{\partial}{\partial y}\left[\mu\left(\frac{\partial v}{\partial z}+\frac{\partial w}{\partial y}\right)\right]\right\} d \Omega d X \\
& -\int_{0}^{X_{t}} \int_{\Omega} T^{a}\left\{\rho \mathbf{V} \cdot \nabla T-\frac{1}{P r} \nabla_{c} \cdot\left(k \nabla_{c} T\right)-(\gamma-1) M_{\infty}^{2} \mu\left[\left(\frac{\partial u}{\partial y}\right)^{2}+\left(\frac{\partial u}{\partial z}\right)^{2}\right]\right\} d \Omega d X \\
& -\int_{0}^{X_{t}} \int_{\Omega} p^{a}\left(p-\frac{\rho T}{\gamma M_{\infty}^{2}} R_{e \lambda}^{2}\right) d \Omega d X \\
& -\int_{0}^{X_{t}} \int_{\Omega} \mu^{a}\left(\mu-T^{b}\right) d \Omega d X \\
& -\int_{0}^{X_{t}} \int_{\Omega} k^{a}(k-\mu) d \Omega d X
\end{aligned}
$$

In the above equations, the control is applied in specified intervals $\left[X_{0}, X_{1}\right]$ only, $\Omega$ is the cross-section domain $[0, \infty] \times\left[z_{1}, z_{2}\right]$ ranging from the wall $(y=0)$ to infinity and from $z_{1}$ to $z_{2}$ in the span-wise direction, $\Gamma$ is the wall boundary line for a given $X, \tau_{w}$ is the wall shear stress, $\tau_{0}$ is a target shear stress (equal to the value corresponding to the Blasius solution), and $\left[X_{s 1}, X_{s 2}\right]$ is the interval where the cost function is defined. The last three integrals in (32) are used to enforce the boundary condition on $v$, which includes the wall transpiration. If we take the directional derivative of the Lagrangian with respect to $\rho$, the result is

$$
\int_{0}^{X_{t}} \int_{\Omega} \rho^{a}(\mathbf{V} \cdot \nabla \delta \rho+\delta \rho \nabla \cdot \mathbf{V})+\delta \rho \mathbf{V} \cdot\left(u^{a} \nabla u+v^{a} \nabla v+w^{a} \nabla w+T^{a} \nabla T\right)-p^{a} \frac{\delta \rho T}{\gamma M_{\infty}^{2}} R_{e \lambda}^{2} d \Omega d X=0
$$




$$
\begin{aligned}
& \int_{0}^{X_{t}} \int_{\Omega} \delta \rho\left[\mathbf{V} \cdot\left(u^{a} \nabla u+v^{a} \nabla v+w^{a} \nabla w+T^{a} \nabla T\right)-p^{a} \frac{T R_{e \lambda}^{2}}{\gamma M_{\infty}^{2}} T-\boldsymbol{V} \cdot \nabla \rho^{a}\right] d \Omega d X \\
& \quad+\left.\int_{\Omega} \rho^{a} u \delta \rho\right|_{0} ^{X_{t}} d \Omega \\
& \quad+\left.\int_{0}^{X_{t}} \int_{\Gamma} \rho^{a}(v+w) \delta \rho\right|_{\Gamma} d \Gamma d X=0
\end{aligned}
$$

The second and third integrals are obtained from integration by parts, respectively, in $\left[0, X_{t}\right]$ and in $\Omega$; assuming arbitrary variations of $\delta \rho$, the first adjoint equation is obtained in the form

$$
\mathbf{V} \cdot\left(u^{a} \nabla u+v^{a} \nabla v+w^{a} \nabla w+T^{a} \nabla T\right)-p^{a} \frac{T R_{e \lambda}^{2}}{\gamma M_{\infty}^{2}}-\mathbf{V} \cdot \nabla \rho^{a}=0 \quad \text { on } \quad\left[0, X_{t}\right] \times \Omega
$$

where $\left.\delta \rho\right|_{\Gamma}=0,\left.\delta \rho\right|_{0} ^{X_{t}}=0$, and $\mathbf{V}^{a}=u^{a} \vec{i}+v^{a} \vec{j}+w^{a} \vec{k}$.

Similarly, we take the directional derivative of the Lagrangian with respect to rest of the state variables $u, v, w, T$ and obtain

$$
\begin{gathered}
\rho\left(\mathbf{V}^{a} \cdot \frac{\partial \mathbf{V}}{\partial x}+T^{a} \frac{\partial T}{\partial x}\right)+2 G_{\Lambda} u v^{a}-\rho \frac{\partial \rho^{a}}{\partial x}-\rho \mathbf{V} \cdot \nabla u^{a}-\left(\mu \nabla_{c} \cdot\left(\nabla_{c} u^{a}\right)+\nabla_{c} \mu \cdot \nabla_{c} u^{a}\right)+\frac{2}{3} \frac{\partial \mu}{\partial x}\left(\nabla_{c} \cdot \mathbf{V}^{a}\right) \\
-\left(\frac{1}{3} \mu \nabla_{c} \cdot \frac{\partial \mathbf{V}^{a}}{\partial x}+\nabla_{c} \mu \cdot \frac{\partial \mathbf{V}^{a}}{\partial x}\right)=0 \quad \text { on } \quad\left[0, X_{t}\right] \times \Omega \\
\rho\left(\mathbf{V}^{a} \cdot \frac{\partial \mathbf{V}}{\partial y}+T^{a} \frac{\partial T}{\partial y}\right)-\rho \frac{\partial \rho^{a}}{\partial y}-\rho \boldsymbol{V} \cdot \nabla v^{a}-\left[\frac{4}{3}\left(\frac{\partial \mu}{\partial y} \frac{\partial v^{a}}{\partial y}+\mu \frac{\partial^{2} v^{a}}{\partial y^{2}}\right)+\frac{\partial \mu}{\partial z} \frac{\partial v^{a}}{\partial z}+\mu \frac{\partial^{2} v^{a}}{\partial z^{2}}+\frac{1}{3} \mu \frac{\partial^{2} w^{a}}{\partial y \partial z}\right. \\
\left.+\frac{\partial \mu}{\partial z} \frac{\partial w^{a}}{\partial y}\right]+\frac{2}{3} \frac{\partial \mu}{\partial y} \frac{\partial w^{a}}{\partial z}=0 \quad \text { on } \quad\left[0, X_{t}\right] \times \Omega \\
\rho\left(\mathbf{V}^{a} \cdot \frac{\partial \mathbf{V}}{\partial z}+T^{a} \frac{\partial T}{\partial z}\right)-\rho \frac{\partial \rho^{a}}{\partial z}-\rho \boldsymbol{V} \cdot \nabla w^{a}-\left[\frac{4}{3}\left(\frac{\partial \mu}{\partial z} \frac{\partial w^{a}}{\partial z}+\mu \frac{\partial^{2} w^{a}}{\partial z^{2}}\right)+\frac{\partial \mu}{\partial y} \frac{\partial w^{a}}{\partial y}+\mu \frac{\partial^{2} w^{a}}{\partial y^{2}}+\frac{1}{3} \mu \frac{\partial^{2} v^{a}}{\partial y \partial z}\right. \\
\left.+\frac{\partial \mu}{\partial y} \frac{\partial v^{a}}{\partial z}\right]+\frac{2}{3} \frac{\partial \mu}{\partial z} \frac{\partial v^{a}}{\partial y}=0 \quad \text { on } \quad\left[0, X_{t}\right] \times \Omega \quad \\
\rho \boldsymbol{V} \cdot \nabla T^{a}+\frac{1}{P r}\left(\nabla_{c} k \cdot \nabla_{c} T^{a}+k \nabla_{c}^{2} T^{a}\right)-p^{a} \frac{\rho R_{e \lambda}^{2}}{\gamma M_{\infty}^{2}}+\mu^{a} b T^{b-1}=0 \quad \text { on } \quad\left[0, X_{t}\right] \times \Omega
\end{gathered}
$$

The initial and boundary conditions associated with the adjoint equations are

$$
\begin{gathered}
\left.\left(\rho^{a}, u^{a}, v^{a}, w^{a}, T^{a}\right)\right|_{X=X_{t}}=(0,0,0,0,0) \text { in } \Omega \\
\left.\left(u^{a}, v^{a}, w^{a}\right)\right|_{\Gamma}=\left\{\begin{array}{l}
\left(\alpha\left(\tau_{w}-\tau_{0}\right), 0,0\right) \text { for } X \in\left[X_{s 0}, X_{s 1}\right] \\
(0,0,0) \text { otherwise }
\end{array}\right.
\end{gathered}
$$

and

$$
\left.\left(\rho^{a}, u^{a}, v^{a}, w^{a}, T^{a}\right)\right|_{Y \rightarrow \infty}=(0,0,0,0)
$$

where $\alpha$ is a constant pre-factor that controls the penalization of the wall shear stress. With the state variables $(\rho, u, v, w, T)$ determined from equations (14)-(17), the adjoint equations (35)-(39) are linear and 
parabolic, and can be solved via a marching procedure in the backward direction, starting from the terminal streamwise location, $X_{t}$, towards the initial streamwise location $X_{0}$. In the present study, the values of the constant factors $\alpha$ and $\sigma$ are set to 1 and 0.1, respectively.

The non time-dependent adjoint variables $p^{a}, \mu^{a}, k^{a}$ are determined in a similar fashion

$$
\begin{gathered}
p^{a}=\frac{\partial v^{a}}{\partial y}+\frac{\partial w^{a}}{\partial z} \\
k^{a}=-\frac{1}{\operatorname{Pr}}\left(\frac{\partial T^{a}}{\partial y} \frac{\partial T}{\partial y}+\frac{\partial T^{a}}{\partial z} \frac{\partial T}{\partial z}\right) \\
\mu^{a}=k^{a}-\left[\nabla_{c} u^{a} \cdot \nabla_{c} u+\frac{2}{3} \frac{\partial v^{a}}{\partial y}\left(2 \frac{\partial v}{\partial y}+\frac{\partial u}{\partial x}+\frac{\partial w}{\partial z}\right)+\frac{2}{3} \frac{\partial w^{a}}{\partial z}\left(2 \frac{\partial w}{\partial z}+\frac{\partial u}{\partial x}+\frac{\partial v}{\partial y}\right)\right. \\
\left.\nabla_{c} u \cdot \frac{\partial \boldsymbol{V}^{a}}{\partial x}+\left(\frac{\partial v^{a}}{\partial z}+\frac{\partial w^{a}}{\partial y}\right)\left(\frac{\partial v}{\partial z}+\frac{\partial w}{\partial y}\right)\right]
\end{gathered}
$$

The control variables are updated using the optimality conditions given by

$$
\sigma\left(v_{w}-\frac{\partial^{2} v_{w}}{\partial x^{2}}\right)+s=0
$$

where $s$ is the Lagrange multiplier corresponding to the transpiration condition. It is obtained from the derivation of the third adjoint equation (37) as result of the integration by parts

$$
s=-\rho^{a} \rho-v^{a} \rho(u+v+w)+\mu\left(\frac{4}{3} \frac{\partial v^{a}}{\partial y}+\frac{\partial v^{a}}{\partial z}-\frac{2}{3} \frac{\partial w^{a}}{\partial z}+\frac{\partial w^{a}}{\partial y}\right)
$$

The control algorithm starts with the solution to the compressible BRE for the uncontrolled boundary layer, followed by the solution to the adjoint compressible BRE (note that the adjoint BRE depend on the solution to the BRE). The difference between the wall shear stress and the original laminar wall shear stress is then compared to a desired value; if the difference is larger than a given threshold then the steepest descent method is utilized to determine the new wall transpiration velocity $v_{w}$. Once these are determined, the loop is repeated, and the calculation finishes when the difference between the wall shear stress and the desired value is less than the threshold.

The adjoint equations (35)-(39) and the associated initial and boundary conditions (40)-(42) are solved numerically on the same grid as the original BRE state equations (14)-(17), and utilizing the same numerical algorithm (as for the BRE equations), except that the marching is preformed backwards, starting from the terminal streamwise location.

Thanks to the robust mathematical model of the NCBRE and the associated numerical algorithm investigated in our previous work, ${ }^{10}$ the overall computational cost of the control algorithm is reduced.

\section{Results and discussion}

In this section, we present the numerical simulation results of the boundary layer optimal control approach. The flow Mach number is set to $M_{\infty}=4, M_{\infty}=5$, and $M_{\infty}=6$ to examine the efficiency of the control approach across different flow conditions (at this point, the control algorithm was found to be unstable at lower supersonic Mach numbers, but work is underway to fix this issue). We introduce flow instabilities using a row of roughness elements located at $0.8 \mathrm{~m}$ from the leading edge. The spanwise separation $\lambda^{*}$ between the roughness elements and the surface curvature radius $r^{*}$ are respectively set to $0.8 \mathrm{~cm}$ and $1.5 \mathrm{~m}$ in the numerical setup. Since we are using wall transpiration to control the flow, the cumulative blowing or suction at the wall is employed such that a zero mass flow rate is maintained to avoid injecting or absorbing mass into/from the flow.

The kinetic energy distribution is calculated according to the characteristic length $L$ as

$$
E(x)=\int_{z_{1}}^{z_{2}} \int_{-\infty}^{\infty}\left[\left|u(x, y, z)-u_{m}(x, y)\right|^{2}+\left|v(x, y, z)-v_{m}(x, y)\right|^{2}+\left|w(x, y, z)-w_{m}(x, y)\right|^{2}\right] d z d y
$$


where $u_{m}(x, y), v_{m}(x, y)$, and $w_{m}(x, y)$ are the spanwise mean components of velocity, and $z_{1}$ and $z_{2}$ are the coordinates of the boundaries in the spanwise direction.

From figure 1, it is clear that the optimal control approach causes a reduction in the the kinetic energy of the flow. The blowing or suction associated with the transpiration velocity control variable are applied as to reduce the momentum of the flow, thanks to the optimal control model, and therefore the kinetic energy of the centrifugal instabilities decreases. The amount of this reduction decreases as the number of iterations increase. However, note that increasing the number of iterations does not necessarily translate to a further reduction in the kinetic energy, on the contrary, this might have an adverse effect on the flow and consequently cause the kinetic energy level to increase. Our trials showed that four iterations are adequate to achieve optimal reduction. We report $28.6 \%, 26.7$, and $31.6 \%$ drop in $E$ respectively achieved for the $M_{\infty}=4, M_{\infty}=5$, and $M_{\infty}=6$ cases when comparing the fourth control iteration maximum energy value to the uncontrolled flow case. In the context of transition from laminar to turbulent flow, the reduction in the kinetic energy of the flow implies that the occurrence of the secondary instabilities leading to transition is delayed, which consequently elongates the laminar region of the boundary layer. The vortex energy growth in figure 3 follows a similar pattern.

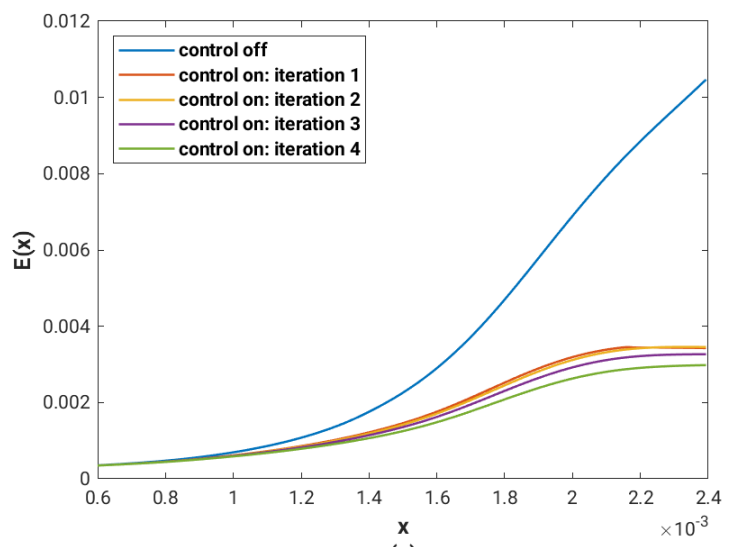

(a)

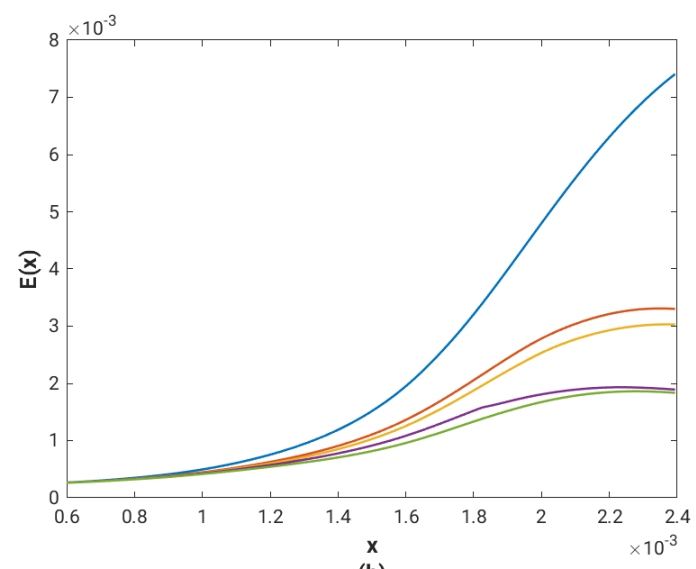

(b)

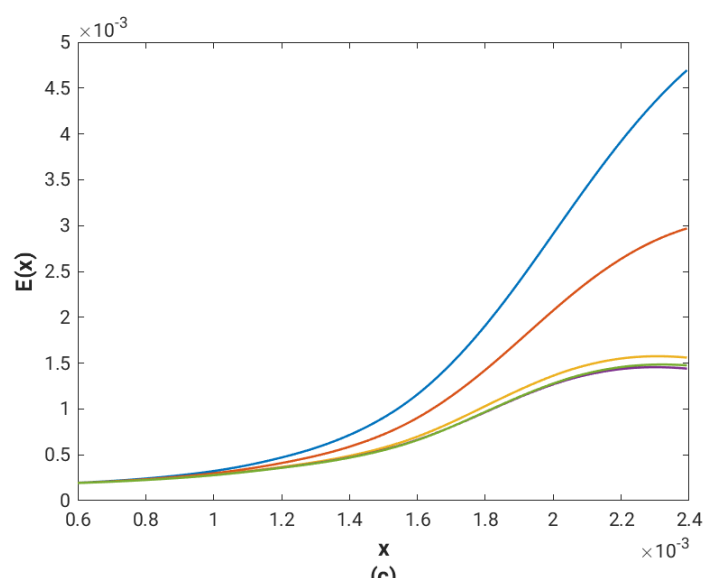

(c)

Figure 2: Vortex energy of the uncontrolled and controlled boundary layer for a) $\mathrm{M}=4, \mathrm{~b}) \mathrm{M}=5, \mathrm{c}) \mathrm{M}=$ 6 . 


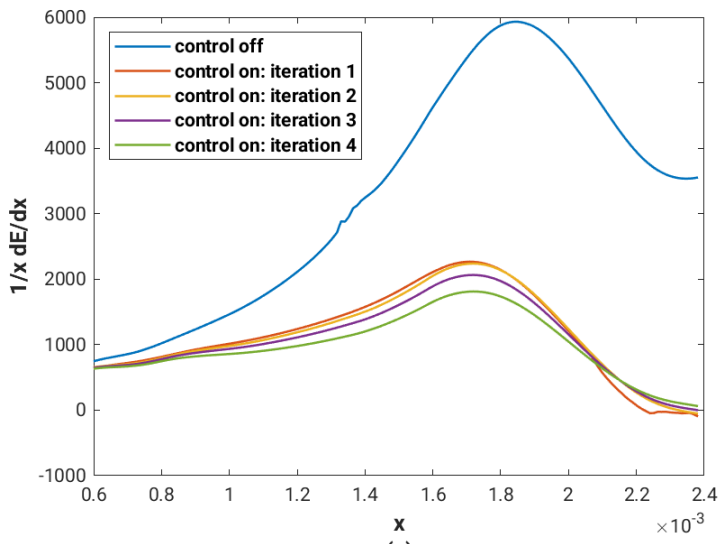

(a)

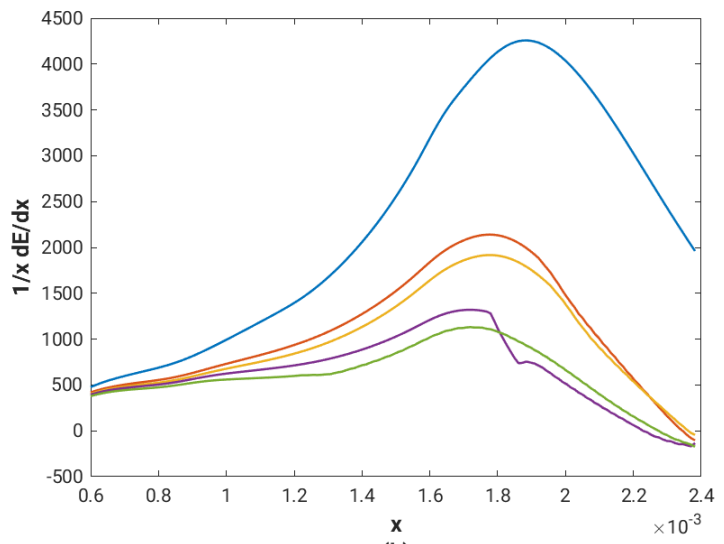

(b)



(c)

Figure 3: Vortex energy growth of the uncontrolled and controlled boundary layer for a) $\mathrm{M}=4$, b) $\mathrm{M}=5$, c) $\mathrm{M}=6$. 
The spanwise averaged wall shear stress is evaluated using the integral

$$
\tau_{w}(x)=\left.\frac{1}{\left(z_{2}-z_{1}\right)} \int_{z_{1}}^{z_{2}} \frac{\partial u}{\partial y}\right|_{y=0}(x, 0, z) d z
$$

The plots in figure 4 show the wall shear stress distribution of the uncontrolled boundary layer and the associated control iterations. The boundary layer wall shear stress decreases as a result of the control approach. As more control iterations are applied, $\tau_{w}$ converges, although not completely, towards the undisturbed case. Taking the undisturbed boundary layer case (e.g., the laminar Blasius solution) as a reference, we report $34.5 \%, 28.5 \%$, and $20 \%$ reduction in $\tau_{w}$ for the $M_{\infty}=4, M_{\infty}=5$, and $M_{\infty}=6$ cases, respectively. The reduction in the wall shear stress directly translates to a reduction in the boundary layer skin friction.

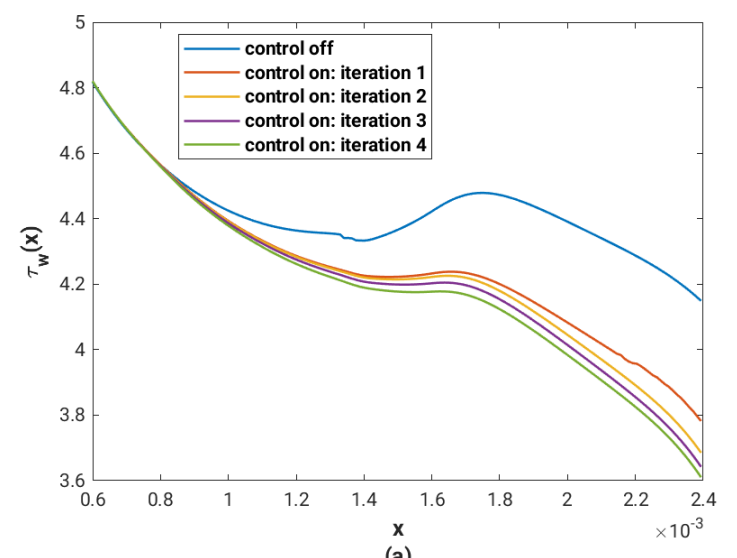

(a)

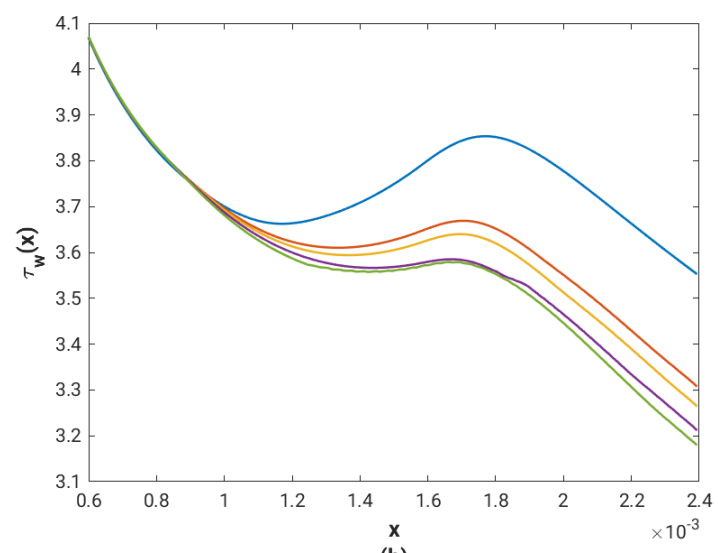

(b)

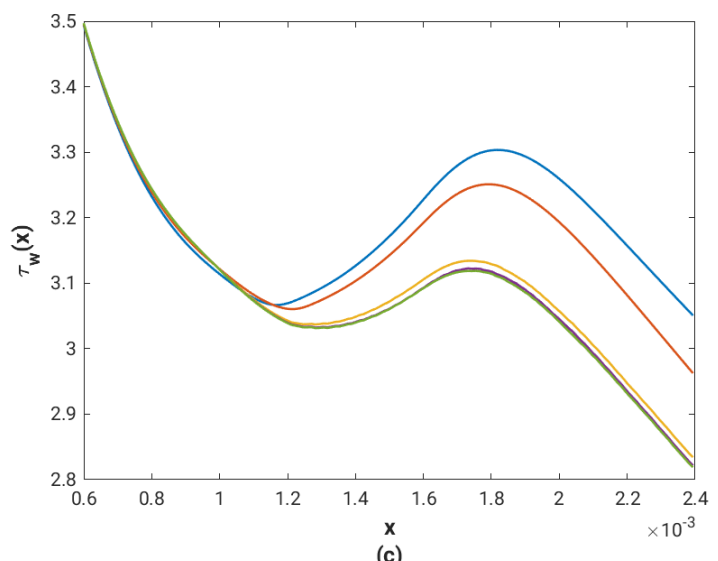

(c)

Figure 4: Wall shear stress of the uncontrolled and controlled boundary layer for a) $\mathrm{M}=4, \mathrm{~b}$ ) $\mathrm{M}=5$, c) $\mathrm{M}$ $=6$.

The streamwise primary instability contours in figure 5 show regions of low and high-speed flow at the same streamwise location. The contours visually show the effect of the control approach on the centrifugal instabilities of the boundary layer. In the case of the uncontrolled instabilities (first contour plot from the top), the Görtler vortices correspond to fully developed mushroom-like structures with alternating low and high-speed streaks in the spanwise direction. However, the shape of the instabilities (especially in the region closest to the wall) is drastically altered as a result of the control approach. The instabilities gradually flatten out as the number of control iterations is increased. This behavior is a direct result of the reduction in the kinetic energy and wall shear stress levels in the boundary layer owing to our control algorithm, as discussed above. 

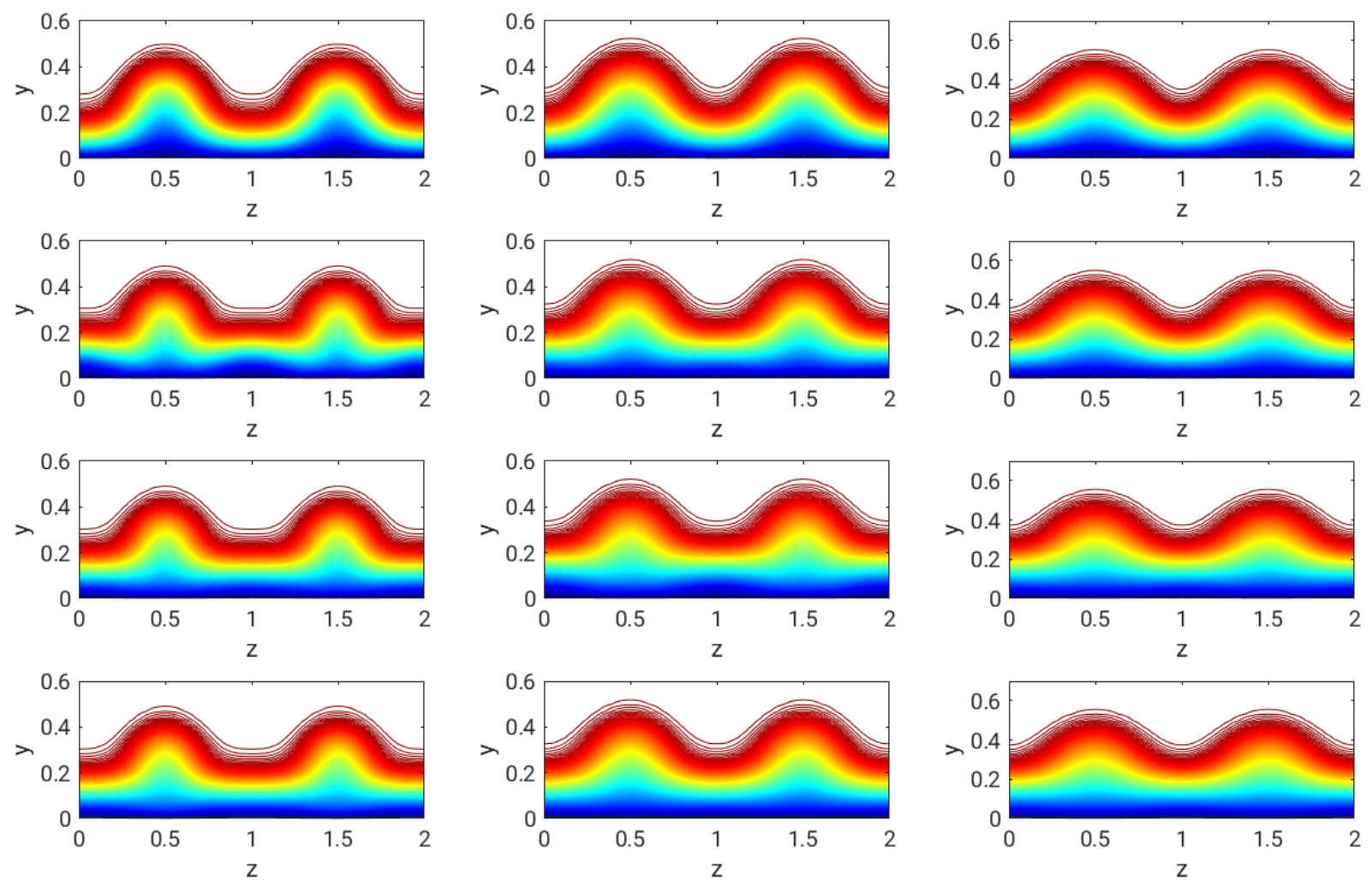

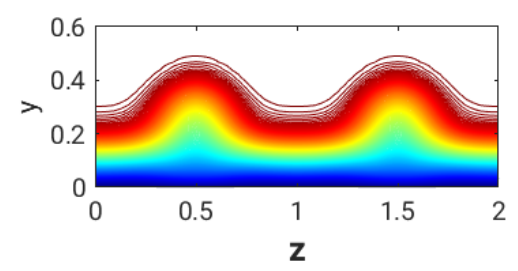

(a)

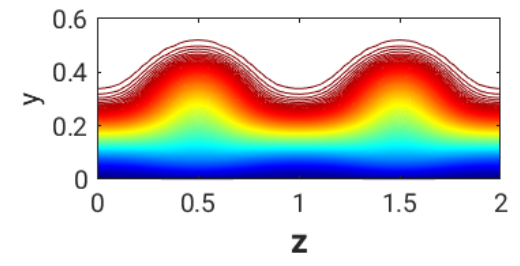

(b)

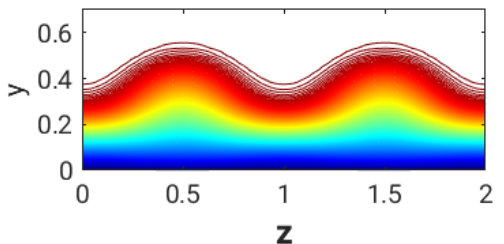

(c)

Figure 5: Streamwise velocity contour plots for a) $\mathrm{M}=4$, b) $\mathrm{M}=5$, c) $\mathrm{M}=6$; from top to bottom, contours respectively represent the uncontrolled flow, $1^{\text {st }}$ control iteration, $2^{\text {nd }}$ control iteration, $3^{\text {rd }}$ control iteration, and $4^{\text {th }}$ control iteration. 


\section{Conclusion}

In this study, we presented an optimal control approach to suppress the growth of the centrifugal instabilities evolving in a compressible boundary layer over a curved surface using the numerical solution to the boundary region equations, a parabolized form of the Navier-Stokes equations, and the optimal control formalism.

The kinetic energy plots of the centrifugal instabilities in figure 2 showed that the optimal control approach induces a significant reduction in $E$ as a result of the blowing or suction associated with the transpiration velocity control variable. Our control approach resulted in $28.6 \%, 26.7 \%$, and $31.6 \%$ reduction in $E$ respectively for the $M_{\infty}=4, M_{\infty}=5$, and $M_{\infty}=6$ cases when comparing the fourth control iteration maximum energy value to the uncontrolled flow case. According to our trials, the number of control iterations should be limited to four iterations to achieve optimal reduction. Applying more control iterations could result in an adverse effect on the flow and consequently cause the kinetic energy to increase. In terms of the transition from laminar to turbulent flow, the reduction in the kinetic energy of the flow implies a longer laminar region of the boundary layer as the secondary instabilities leading to transition are delayed.

The control approach also induced a significant reduction in the wall shear stress level of the boundary layer flow, as illustrated in figure 4. Though we did not achieve a complete convergence, the wall shear stress corresponding to the controlled boundary layer approached the undisturbed boundary layer counterpart due to the control iterations. Considering the undisturbed case as a reference (e.g., the laminar Blasius solution), we achieved $34.5 \%, 28.5 \%$, and $20 \%$ reduction in $\tau_{w}$ for the $M_{\infty}=4, M_{\infty}=5$, and $M_{\infty}=6$ cases, respectively. The reduction in the wall shear stress directly translates to a reduction in the boundary layer skin friction.

The crossflow contours of the streamwise primary instability in figure 5 visually captured the effect of the control approach on the centrifugal instabilities of the boundary layer. Applying more control iterations gradually flattened out the instabilities (especially near the wall). This finding goes hand in hand with the reduction in the kinetic energy and wall shear stress levels in the boundary layer.

The preliminary results presented in this study all demonstrate the efficiency of our optimal control approach in suppressing the growth rate of the streamwise vortex for high supersonic Mach number. Work is underway to fix the stability and convergence issues of the algorithm for lower supersonic Mach numbers.

\section{References}

${ }^{1}$ Bewley, T., and Moin, P. (1994) Optimal control of turbulent channel flows, ASME DE, Vol. 75, pp. 221-227.

${ }^{2}$ Boiko, A.V., Ivanov, A.V., Kachanov, Y.S. and Mischenko, D.A. (2010) Steady and unsteady Görtler boundary-layer instability on concave wall, Eur. J. Mech. B - Fluids, Vol. 29, pp. 61-83.

${ }^{3}$ Cathalifaud, P., and Luchini, P. (2000) Algebraic growth in boundary layers: optimal control by blowing and suction at the wall, Eur. J. Mech. B - Fluids. Vol. 19, pp. 469-490.

${ }^{4}$ Cherubini, S., Robinet, J.-C., and De Palma, P. (2013) Nonlinear control of unsteady finite-amplitude perturbations in the Blasius boundary-layer flow, J. Fluid Mech.. Vol. 737, pp. 440-465.

${ }^{5}$ Choi, H., Moin, P., and Kim, J. (1994) Active turbulence control for reduction in wall-bounded flows, J. Fluid Mech.. Vol. 262, pp. $75-110$.

${ }^{6}$ Choudhari, M. and Fischer, P. (2005) Roughness induced Transient Growth, AIAA Paper 2005-4765.

${ }^{7}$ Corbett, P. and Bottaro, A. (2001) Optimal Control of Nonmodal Disturbances in Boundary Layers, Theoret. Comput. Fluid Dynamics. Vol. 15, pp. 65-81.

${ }^{8}$ Depmsey, L.D., Hall, P. and Deguchiu, K. (2017) The excitation of Gortler vortices by free stream coherent structures, J. Fluid Mech., Vol. 826, pp. 60-96.

${ }^{9}$ Es-Sahli, O., Sescu, A., Afsar, M., Hattori, Y., and Hirota, M. (2021) Effect of localized wall cooling or heating on streaks in high-speed boundary layers, AIAA Scitech, AIAA 2021-0853.

${ }^{10}$ Es-Sahli, O., Sescu, A., Afsar, M., and Hattori, Y. (2021) Investigation of Görtler vortices in high-speed boundary layers via an efficient numerical solution to the non-linear boundary region equations, Theoret. Comput. Fluid Dynamics, in press.

${ }^{11}$ Gad-el-Hak, M. (1989) Flow Control, Applied Mechanics Review. Vol. 42, pp. 261-293.

${ }^{12}$ Goldstein, M., Sescu, A., Duck, P. and Choudhari, M. (2010) The Long Range Persistence of Wakes behind a Row of Roughness Elements, J. Fluid Mech., Vol. 644, pp. 123-163.

${ }^{13}$ Goldstein, M., Sescu, A., Duck, P. and Choudhari, M. (2011) Algebraic/transcendental Disturbance Growth behind a Row of Roughness Elements, J. Fluid Mech., Vol. 668, pp. 236-266.

${ }^{14}$ Goldstein, M., Sescu, A., Duck, P. and Choudhari, M. (2016) Nonlinear wakes behind a row of elongated roughness elements, J. Fluid Mech., Vol. 796, pp. 516-557.

${ }^{15}$ Goldstein, M., and Sescu, A. (2008) Boundary-layer transition at high free-stream disturbance levels - beyond Klebanoff modes. J. Fluid Mech., Vol. 613, pp. 95-124. 
${ }^{16}$ Görtler, H . (1941) Instabilita-umt laminarer Grenzchichten an Konkaven Wanden gegenber gewissen dreidimensionalen Storungen, ZAMM, Vol. 21, pp. 250-52; english version: NACA Report 1375 (1954)

${ }^{17}$ Gunzburger, M. (2000) Adjoint Equation-Based Methods for Control Problems in Incompressible, Viscous Flows, Flow, Turbulence and Combustion. Vol. 65, pp. 249.

${ }^{18}$ Hall, P. (1982) Taylor-Görtler vortices in fully developed or boundary-layer flows: linear theory, J. Fluid Mech., Vol. 124, pp. 475-494.

${ }^{19}$ Hall, P. (1983) The linear development of Görtler vortices in growing boundary layers, J. Fluid Mech., Vol. 130, pp. $41-58$.

${ }^{20}$ Hall, P. and Horseman. N. (1991) The linear inviscid secondary instability of longitudinal vortex structures in boundary layers. J. Fluid Mech., Vol. 232, pp. 357-375.

${ }^{21}$ Högberg, M., Bewley, T.R., Henningson, D.S. (2003) Relaminarization of $R e_{\tau}=100$ turbulence using gain scheduling and linear state feedback control, Phys. Fluids, Vol. 15, pp. 3572.

22 Jacobson, S.A., and Reynolds, W.C. (1998) Active control of streamwise vortices and streaks in boundary layers J. Fluid Mech., Vol. 360, pp. 179-211.

${ }^{23}$ Joslin, R., Gunzburger, M., Nicolaides, R., Erlebacher, G., and Hussaini, M. (1197) Self-Contained Automated Methodology for Optimal Flow Control, AIAA J.. Vol. 35, pp. 816-824.

${ }^{24}$ Kendall, J.M. (1998) Experiments on boundary-layer receptivity to freestream turbulence, AIAA Paper 2004-2335.

${ }^{25} \mathrm{Kim}$, J. (2003) Control of turbulent boundary layers, Phys. Fluids. Vol. 15, pp. 1093.

${ }^{26}$ Koumoutsakos, P. (1997) Active control of vortex-wall interactions, Phys. Fluids. Vol. 9, pp. 3808.

${ }^{27}$ Koumoutsakos, P. (1999) Vorticity flux control for a turbulent channel flow, Phys. Fluids. Vol. 11, pp. 248.

${ }^{28}$ Landahl, M.T. (1980) A note on an algebraic instability of inviscid parallel shear flows. J. Fluid Mech., Vol. 98, pp. $243-251$

${ }^{29}$ Lee, C., Kim, J., and Choi, H. (1998) Suboptimal control of turbulent channel flow for drag reduction, J. Fluid Mech.. Vol. 358, pp. 245-258.

${ }^{30}$ Leib, S.J., Wundrow, W., and Goldstein, M. (1999) Effect of free-stream turbulence and other vortical disturbances on a laminar boundary layer. J. Fluid Mech., Vol. 380, pp. 169-203.

${ }^{31} \mathrm{Li}$, F., and Malik, M. (1995) Fundamental and subharmonic secondary instabilities of Görtler vortices, J. Fluid Mech., Vol. 297, pp. 77-100.

${ }^{32}$ Li, F., Choudhari, M., Chang, C.-L., Greene, P., and Wu, M. (2010) Development and Breakdown of Gortler Vortices in High Speed Boundary Layers", 48th AIAA Aerospace Sciences Meeting Including the New Horizons Forum and Aerospace Exposition, Aerospace Sciences Meetings.

${ }^{33}$ Lu, L., Agostini, L., Ricco, P., \& Papadakis, P., (2014) Optimal state feedback control of streaks and Gortler vortices induced by free-stream vortical disturbances, UKACC International Conference on Control. Loughborough, U.K.

${ }^{34}$ Luchini, P., and Bottaro, A. (2014) Adjoint Equations in Stability Analysis, Annu. Rev. Fluid Mech.. Vol. 46, pp. 493-517.

${ }^{35}$ Lundell, F., and Alfredsson, P.H. (2003) Experiments on control of streamwise streaks, Eur. J. Mech. (B/Fluids). Vol. 22 , pp. $1279-1290$.

${ }^{36}$ Malik, M.R., and Hussaini, M.Y. (1990) Numerical simulation of interactions between Gortler vortices and TollmienSchlichting waves. J. Fluid Mech., Vol. 210, pp. 183-199.

${ }^{37}$ Marensi, E., Ricco, P., and Wu, X. (2017) Nonlinear unsteady streaks engendered by the interaction of free-stream vorticity with a compressible boundary layer. J. Fluid Mech.. Vol. 817, pp. 80-121.

${ }^{38}$ Matsubara, M., and Alfredsson, P.H. (2001) Disturbance growth in boundary layers subjected to free stream turbulence, J. Fluid Mech., Vol. 430, pp. 149.

${ }^{39}$ Myose, R. Y., and Blackwelder, R. F. (1995) Control of streamwise vortices using selective suction, AIAA Journal, Vol. 33, pp 1076-1080.

${ }^{40}$ Pamies, M., Garnier, E., Merlen, A., and Sagaut, P. (2007) Response of a spatially developing turbulent boundary layer to active control strategies in the framework of opposition control, Phys. Fluids. Vol. 19, pp. 108102.

${ }^{41}$ Papadakis, G., Lu,L., and Ricco, P. (2016) Closed-loop control of boundary layer streaks induced by free-stream turbulence, Phys. Rev. Fluids. Vol. 1, pp. 043501.

${ }^{42}$ Ren, J., and Fu, S. (2017) Secondary instabilities of Gortler vortices in high-speed boundary layer flows, J. Fluid Mech., Vol. 781 , pp. 388-421.

${ }^{43}$ Ricco, P. (2011) Laminar streaks with spanwise wall forcing, Phys. Fluids, Vol. 23, pp. 064103.

${ }^{44}$ Ricco, P. (2006) Response of a compressible laminar boundary layer to freestream turbulence. PhD thesis, University of London.

${ }^{45}$ Saric, W.S. (1994) Görtler Vortices, Annu. Rev. Fluid Mech., Vol. 26, pp. 379-409.

${ }^{46}$ Schneider, P. S. (2001) Effects of high-speed tunnel noise on laminar-turbulent transition, J. Spacecr. Rockets, Vol 38, pp 323-333.

${ }^{47}$ Sescu, A., Pendyala, R., and Thompson, D. (2014) On the Growth of Görtler Vortices Excited by Distributed Roughness Elements, AIAA Paper 2014-2885.

${ }^{48} \mathrm{Sescu}$, A., and Thompson, D. (2015) On the Excitation of Görtler Vortices by Distributed Roughness Elements, Theoret. Comput. Fluid Dynamics, Vol. 29, pp. 67-92.

${ }^{49}$ Sescu, A., Taoudi, L., Afsar, M., and Thompson D. (2016) Control of Gortler Vortices by Means of Surface Streaks, AIAA Paper 2016-3950.

${ }^{50}$ Sescu, A., Taoudi, L. \& Afsar, M. (2018) Iterative control of Gortler vortices via local wall deformations, Theoret. Comput. Fluid Dynamics, Vol. 32, pp. 63-72.

${ }^{51} \mathrm{Sescu}, \mathrm{A}$., and Afsar, M. (2018) Hampering Gortler vortices via optimal control in the framework of nonlinear boundary region equations, J. Fluid Mech., Vol. 848, p. 5-41. 
${ }^{52}$ Sescu, A., Alaziz, R., and Afsar, M.Z. (2019) Effect of Wall Transpiration and Heat Transfer on Nonlinear Gortler Vortices in High-speed Boundary Layers, AIAA Journal, doi.org/10.2514/1.J057330

${ }^{53}$ Stroh, A., Frohnapfel, B., Schlatter, P., and Hasegawa, Y. (2015) A comparison of opposition control in turbulent boundary layer and turbulent channel flow, Phys. Fluids. Vol. 27, pp. 075101.

${ }^{54}$ Swearingen, J.D. and Blackwelder, R.F. (1987) The growth and breakdown of streamwise vortices in the presence of a wall. J. Fluid Mech., Vol. 182, pp. 255-290.

${ }^{55}$ Westin, K.J.A., Boiko, A.V., Klingmann, B.J.B., Kozlov, V.V., and Alfredsson, P.H. (1994) Experiments in a boundary layer subjected to free stream turbulence. part 1. Boundary layer structure and receptivity. J. Fluid Mech., Vol. 281 , pp. 193-218.

${ }^{56}$ White, E.B. (2002) Transient growth of stationary disturbances in a flat plate boundary layer, Phys. Fluids, Vol. 14, pp. 4429-4439.

${ }^{57}$ White, E.B., Rice, J.M. and Ergin, F.G. (2005) Receptivity of stationary transient disturbances to surface roughness, Phys. Fluids, Vol. 17, pp. 064109.

${ }^{58} \mathrm{Wu}, \mathrm{X}$, Zhao, D. and Luo, J (2011) Excitation of steady and unsteady Görtler vortices by free-stream vortical disturbances, J. Fluid Mech., Vol. 682, pp. 66-100.

${ }^{59} \mathrm{Wu}, \mathrm{X}$. and Choudhari, M. (2011) Linear and nonlinear instabilities of a blasius boundary layer perturbed by streamwise vortices. Part 2. Intermittent instability induced by long wavelength Klebanoff modes. J. Fluid Mech.. Vol. 483, pp. 249-286.

${ }^{60}$ Xiao, D., and Papadakis, G. (2017) Nonlinear optimal control of bypass transition in a boundary layer flow, Phys. Fluids. Vol. 29, pp. 054103.

${ }^{61} \mathrm{Xu}$, D., Zhang, Y., and Wu, X. (2017) Nonlinear evolution and secondary instability of steady and unsteady Gortler vortices induced by free-stream vortical disturbances, J. Fluid Mech., Vol. 829, pp. 681-730.

${ }^{62}$ Zaki, T.A., and Durbin, P. (2005) Mode interaction and the bypass route to transition, J. Fluid Mech., Vol. 531, pp. 85-111.

${ }^{63}$ Zuccher, S., Luchini, P., and Bottaro, A. (2004) Algebraic growth in a blasius boundary layer: optimal and robust control by mean suction in the nonlinear regime, Eur. J. Mech. B - Fluids. Vol. 513, pp. 135-160. 